University of Nebraska - Lincoln

DigitalCommons@University of Nebraska - Lincoln

Papers in Veterinary and Biomedical Science

Veterinary and Biomedical Sciences,

Department of

February 2000

\title{
Virus-Induced Neuronal Apoptosis Blocked by the Herpes Simplex Virus Latency-Associated Transcript
}

\author{
Guey-Chuen Perng \\ Angeles, $C A$ \\ Clinton J. Jones \\ University of Nebraska - Lincoln, cjones2@unl.edu \\ Janice Ciacci-Zanella \\ University of Nebraska - Lincoln \\ Melissa Stone \\ University of Nebraska - Lincoln \\ Gail A. Henderson \\ University of Nebraska - Lincoln, ghenderson2@unl.edu \\ See next page for additional authors \\ Follow this and additional works at: https://digitalcommons.unl.edu/vetscipapers \\ Part of the Veterinary Medicine Commons
}

Ophthalmology Research Laboratories, Cedars-Sinai, Medical Center Burns \& Allen Research Institute, Los

Perng, Guey-Chuen; Jones, Clinton J.; Ciacci-Zanella, Janice; Stone, Melissa; Henderson, Gail A.; Yukht, Ada; Slanina, Susan M.; Hofman, Florence M.; Ghiasi, Homayon; Nesburn, Anthony B.; and Wechsler, Steven L., "Virus-Induced Neuronal Apoptosis Blocked by the Herpes Simplex Virus Latency-Associated Transcript" (2000). Papers in Veterinary and Biomedical Science. 87.

https://digitalcommons.unl.edu/vetscipapers/87

This Article is brought to you for free and open access by the Veterinary and Biomedical Sciences, Department of at DigitalCommons@University of Nebraska - Lincoln. It has been accepted for inclusion in Papers in Veterinary and Biomedical Science by an authorized administrator of DigitalCommons@University of Nebraska - Lincoln. 


\section{Authors}

Guey-Chuen Perng, Clinton J. Jones, Janice Ciacci-Zanella, Melissa Stone, Gail A. Henderson, Ada Yukht, Susan M. Slanina, Florence M. Hofman, Homayon Ghiasi, Anthony B. Nesburn, and Steven L. Wechsler 


\title{
Virus-Induced Neuronal Apoptosis Blocked by the Herpes Simplex Virus Latency-Associated Transcript
}

\author{
Guey-Chuen Perng, ${ }^{1}$ Clinton Jones, ${ }^{2}$ Janice Ciacci-Zanella, ${ }^{2}$ \\ Melissa Stone, ${ }^{2}$ Gail Henderson, ${ }^{2}$ Ada Yukht, ${ }^{1}$ Susan M. Slanina, ${ }^{1}$ \\ Florence M. Hofman, ${ }^{3}$ Homayon Ghiasi, ${ }^{1,4}$ \\ Anthony B. Nesburn, ${ }^{1,4}$ Steven L. Wechsler ${ }^{1,4 *}$
}

\begin{abstract}
Latent infections with periodic reactivation are a common outcome after acute infection with many viruses. The latency-associated transcript (LAT) gene is required for wild-type reactivation of herpes simplex virus (HSV). However, the underlying mechanisms remain unclear. In rabbit trigeminal ganglia, extensive apoptosis occurred with $L A T^{-}$virus but not with $L A T^{+}$viruses. In addition, a plasmid expressing LAT blocked apoptosis in cultured cells. Thus, LAT promotes neuronal survival after HSV-1 infection by reducing apoptosis.
\end{abstract}

After primary infection of the eye, herpes simplex virus-type 1 (HSV-1) establishes a lifelong latent infection in neurons of the trigeminal ganglia (TGs), with sporadic periods of reactivation and recurrent disease. Recurrent ocular HSV (HSV-1 and HSV-2) is a leading cause of corneal blindness resulting from an infectious agent. Recurrent genital HSV is a serious sexually transmitted disease. Latent HSV infections affect 70 to $90 \%$ of adults.

During latency, a single viral gene-the $L A T$ gene - is abundantly transcribed $(1,2)$. $L A T$ is essential for the efficient reactivation of HSV from latency (3). The primary LAT is 8.3 $\mathrm{kb}$ and overlaps the important immediate early gene ICPO in an antisense direction. Thus, it was proposed that $L A T$ may function through an antisense mechanism $(1,2)$. However, the first $1.5 \mathrm{~kb}$ of LAT alone is sufficient for wildtype levels of spontaneous reactivation (4), and this region does not overlap any known HSV-1 gene. $L A T$ may enhance the establishment or maintenance of latency $(5-8)$, thereby increasing the pool of latently infected neurons, which

${ }^{1}$ Ophthalmology Research Laboratories, Cedars-Sinai Medical Center Burns \& Allen Research Institute, 8700 Beverly Boulevard, Los Angeles, CA 90048, USA. ${ }^{2}$ Department of Veterinary and Biomedical Sciences, Center for Biotechnology, University of Nebraska, Lincoln NE 68583, USA. ${ }^{3}$ Department of Pathology, University of Southern California School of Medicine, Los Angeles, CA 90025, USA. ${ }^{4}$ Department of Ophthalmology, UCLA School of Medicine, Los Angeles, CA 90024, USA.

*To whom correspondence should be addressed. Email: Wechsler@CSMC.edu in turn results in increased levels of spontaneous and/or induced reactivation (9). Although studies with one $L A T^{-}$mutant have suggested that a $L A T$-related function may suppress productive-cycle gene expression during acute and latent infection of mouse trigeminal ganglia $(10,11)$, no evidence was presented to show that $L A T$ caused these effects directly, rather than through a pleiotropic effect. We recently reported on a mutant containing a partial deletion of $L A T$ in which neurovirulence was increased (12). This finding suggested that $L A T$ might protect neurons from being killed by HSV-1, thereby allowing HSV-1 to establish latency in more neurons.

To determine whether neurons were being protected by $L A T$, we used dLAT2903, a $L A T$ null mutant derived from the McKrae strain of HSV-1 (3). This mutant contains a deletion that includes the $L A T$ promoter and the $5^{\prime}$ half of the stable 2-kb $L A T$; this deletion (nucleotides -161 to +1667 ) results in the absence of $L A T$ RNAs and does not overlap or interfere with the ICPO transcript. Like most LAT mutants, dLAT2903 has no known deficits other than being impaired for reactivation from latency. Thus, dLAT2903 is wild type for replication in mouse and rabbit eyes, HSV-1-induced eye disease, replication in TGs, and neurovirulence (3). However, with dLAT2903, large numbers of neurons positive for TUNEL (terminal deoxynucleotidyl transferase-mediated deoxyuridine triphosphate nick-end labeling) were seen in rabbit TGs on day 7 after infection (Table 1; $70 \%$ of sections, $100 \%$ of TGs), whereas in this experiment TUNEL-positive cells were not de- 
tected in TGs from rabbits infected with wildtype McKrae. Only small amounts of TUNELpositive cells were detected in TGs from dLAT2903R, a rescued virus in which the deleted $L A T$ region was restored. The results for dLAT2903-infected rabbits were significantly different from those for the wild typeinfected and dLAT2903R-infected rabbits $[P<0.001$ for sections, $P<0.05$ for TGs; analysis of variance (ANOVA), Tukey comparison test for peak days of apoptosis]. Representative photomicrographs are shown in Fig. 1.

More rabbits were infected (five rabbits per group) and killed on day 7 after infection to study additional aspects of enhanced apoptosis in rabbits infected with the $L A T^{-}$virus (Table 2 ). Fifty sections per group (five sections from each of $10 \mathrm{TGs}$ ) were examined. Extensive apoptosis ( $>25 \%$ TUNEL-positive neurons) was observed in $66 \%$ of the sections from dLAT2903 $\left(L A T^{-}\right)$-infected rabbits, versus only $4 \%$ of the sections from uninfected rabbits or rabbits infected with wild-type McKrae or dLAT2903R $(P<0.001$; ANOVA, Tukey posttest). When all TGs with extensive apoptosis in at least one section were counted (Table 2), $90 \%$ of TGs from rabbits infected with dLAT2903 met this criterion, versus only 10 to $20 \%$ of TGs from uninfected rabbits or rabbits infected with wild-type McKrae or dLAT2903R $(P<0.01)$.

When we counted all TG sections with any amount of apoptosis in neurons, this threshold was met by $74 \%$ of the sections from rabbits infected with dLAT2903; this was significantly higher than for TGs from uninfected rabbits or rabbits infected with wild-type McKrae or dLAT2903R $(P<0.001)$ (Table 2$)$. All 10 TGs from rabbits infected with dLAT2903 were positive according to this criterion, whereas only 5 of 10 TGs from rabbits infected with wild-type McKrae or dLAT2903R were positive $(P=0.03)$ (Table 2$)$. The sporadic low levels of apoptosis detected in rabbit TGs in- fected with wild-type McKrae were expected because HSV-1 can induce apoptosis (13-16).

The results of these TUNEL assays suggest that apoptosis was more likely, by a factor of 2 to 16 , in rabbit TGs infected with the $L A T^{-}$ mutant. Because HSV-1 can induce apoptosis in infected tissue culture cells (13-16), we suspect that the apoptotic neurons detected in TGs by TUNEL were a direct result of the HSV-1 infection. However, because on day 7 (the time of peak apoptosis) viral antigens can no longer be detected in these TGs by immunofluorescent staining (17), it is possible, but unlikely, that some of the apoptosis may be due to a bystander effect.

A newly devised method for detection of apoptosis in tissue sections was used to confirm the TUNEL results. TG sections obtained 7 days after infection were stained with an antibody to poly(ADP-ribose) polymerase (PARP) (Anti-PARP p85 fragment pAb kit; Promega, Madison, WI), as described by the manufacturer. Anti-PARP p85 detects caspase-3 cleavage fragments of PARP, a hallmark of apoptosis (18-20). Representative photomicrographs are shown in Fig. 2. Extensive cleavage of PARP was detected in five of six TGs from $L A T^{-}$infected rabbits ( $>25 \%$ of neurons) but in none of the four TGs from wild-type-infected rabbits $\left(P=0.048\right.$, Fisher exact test). All six $L A T^{-}$ TGs contained detectable levels of cleaved PARP staining, versus only one of four TGs from rabbits infected with wild-type virus $(P=$ 0.03 ). To further confirm the similarity of the TUNEL and anti-PARP p85 results, we prepared seven pairs of sections, each pair from a different TG. One section of each pair was stained for TUNEL; the other section was stained for PARP p85. A correlation was seen between the percentage of positively stained neurons on the TUNEL and anti-PARP p85 sections $\left(R^{2}=0.62, P=0.04\right.$; linear regression; $P<0.05$ indicates that the $R^{2}$ value was unlikely to have occurred by chance). Confirmation of the TUNEL results by anti-PARP p85 staining indicates that most of the TUNELpositive cells were due to apoptosis rather than another mechanism of cell death or reaction with viral DNA ends. Thus, LAT appeared to decrease HSV-1-induced apoptosis in rabbit

Table 1. Apoptosis in rabbit TGs. Rabbits were infected with wild-type HSV-1 McKrae, dLAT2903 (LAT-), or dLAT2903R $\left(L A T^{+}\right)$in both eyes $\left(2 \times 10^{5}\right.$ plaque-forming units per eye), as described (3). On the days indicated after infection, two rabbits were killed per group. TGs were harvested and sectioned, and five sections per TG (20 sections per group at each time point) were stained by the TUNEL assay that detects DNA ends produced during apoptosis (Klenow-FragEL DNA fragmentation detection kit; Oncogene, Cambridge, MA) as described by the manufacturer. Sections were read by light microscopy by two individuals who had no knowledge of the groups and were considered positive for apoptosis if one or more neurons were clearly positive. A TG was considered positive if one or more of its sections were positive. For each TG, the sections used were obtained from different areas spanning the TG.

\begin{tabular}{lrrrr}
\hline \multicolumn{1}{c}{ Criterion } & Day 3 & Day 7 & Day 10 & Day 14 \\
\hline Sections with apoptotic neurons (\%) & & & & \\
$\quad$ dLAT2903 & 5 & 70 & 10 & 5 \\
dLAT2903R & 0 & 0 & 20 & 5 \\
McKrae & 0 & 0 & 0 & 0 \\
TGs with apoptotic neurons (\%) & & & 25 & 25 \\
$\quad$ dLAT2903 & 0 & 100 & 25 & 25 \\
dLAT2903R & 0 & 25 & 0 & 0 \\
McKrae & 0 & 0 & \\
\hline
\end{tabular}

Fig. 1. Representative photomicrographs of TUNEL-stained TG sections (upper and lower rows represent two different experiments). Rabbits were infected, then killed 7 days after infection; TGs were harvested and sections were stained for apoptosis by TUNEL, as described in Table 1. (A) and (D), uninfected; (B) and (E), McKrae-infected; (C) and (F), dLAT2903 (LAT ${ }^{-}$infected. In (A) to (C), positive staining is brown, counterstaining is blue-green; magnification, $\sim \times 150$. In (D) to (F), positive staining is brown, counterstaining is green; magnification, $\sim \times 300$. In (C), nonneuronal cells (apparently infiltrating immune cells), as well as neurons, show strong staining for apoptosis. In (F), brown dots in the nucleus of large neurons indicate a positive signal.
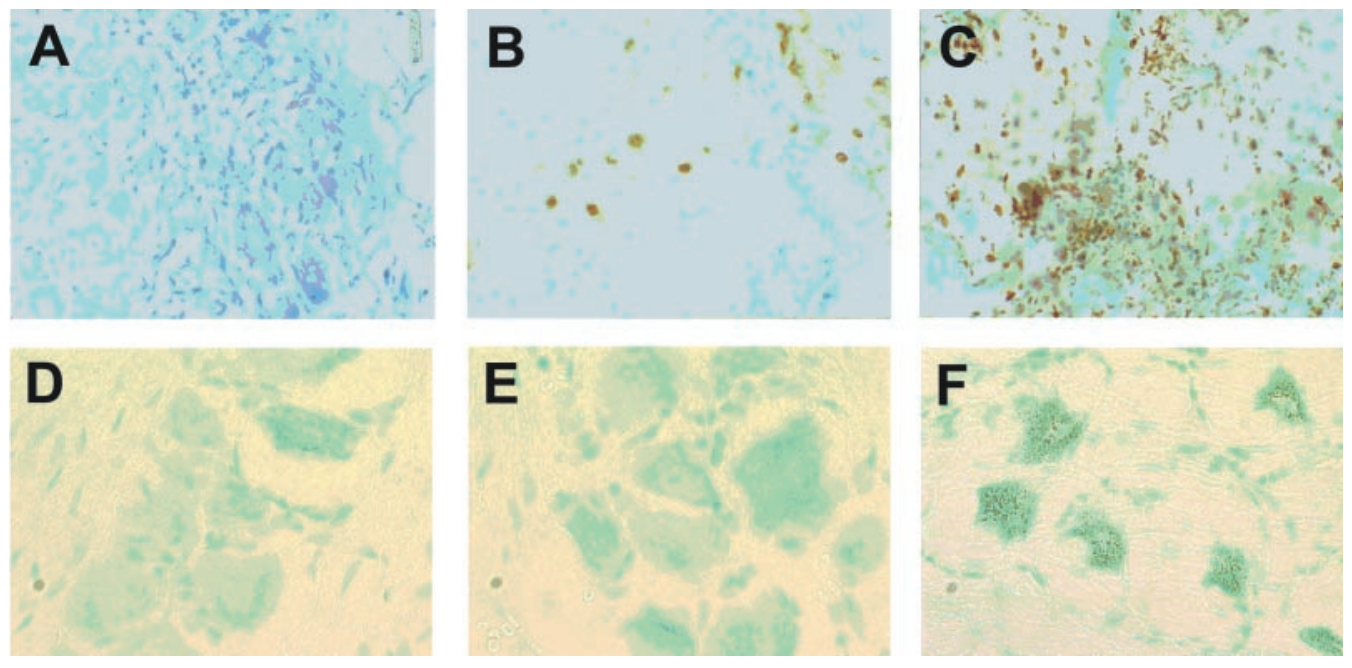


\section{REPORTS}

Fig. 2. Photomicrographs of TG sections stained with anti-PARP p85 (magnification, $\sim \times 300$ ). Rabbits were infected, then killed 7 days after infection; TGs were harvested and sections were stained for apoptosis using the Anti-PARP p85 fragment $P A b$ kit (Promega). Brown dots in the nucleus and cytoplasm of large neurons indicate positive staining. Counterstaining is bluegreen. Representative photomicrographs are shown. (A) Uninfected cells; (B) wild-type McKrae-infected cells; (C) dLAT2903 (LAT ${ }^{-}$)-infected cells.
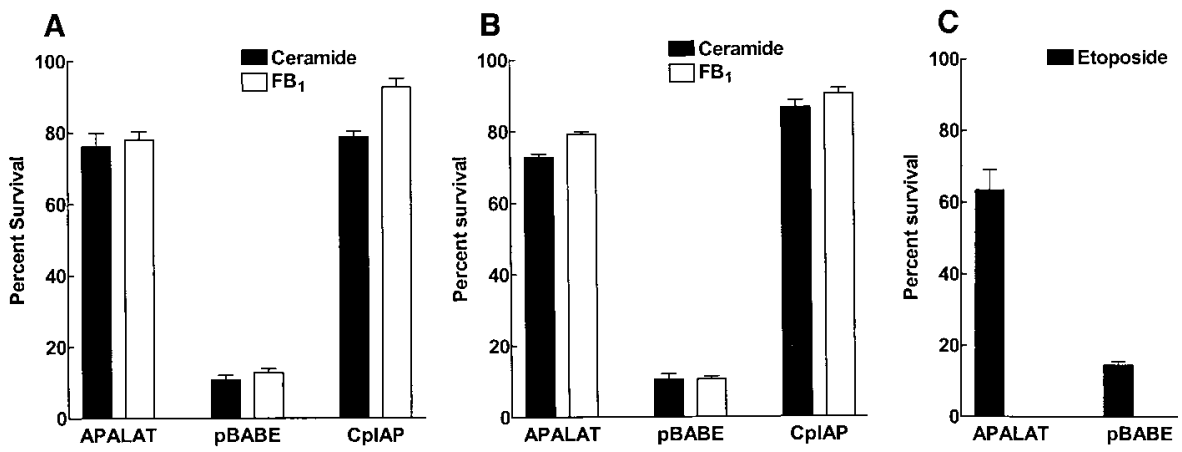

Fig. 3. In vitro inhibition of apoptosis by a LAT plasmid. IMR-90 cells (A), CV-1 cells (B), or neuro-2A cells (C) were cotransfected with $1 \mu \mathrm{g}$ of PCMV- $\beta$-gal (a $\beta$-galactosidase expression plasmid) and $5 \mu \mathrm{g}$ of APALAT (5). APALAT contains an Apa I-Apa I LAT restriction fragment (LAT nucleotides 301 to 2659) inserted into PBABE Puro, a mammalian expression vector containing a retrovirus LTR, Poly(A) addition signals, and the puromycin resistance gene regulated by the SV40 early promoter. Procedures for calcium phosphate transfection and maintenance of cultures were as described (26). Twenty-four hours after transfection, $10 \mu \mathrm{M} \mathrm{C}_{6}$-ceramide (Calbiochem, San Diego, CA) (23-25) or $25 \mu \mathrm{M}$ fumonisin $\mathrm{B}_{1}$ $\left(\mathrm{FB}_{1}\right)(26,27)$ were added to IMR-90 and CV-1 cultures, and $15 \mu \mathrm{M}$ etoposide (Sigma) was added to neuro-2A cultures. After 48 hours, $\beta$-gal ${ }^{+}$cells were identified by staining the fixed cells with Bluo-gal (Gibco-BRL) for 24 hours; $\beta$-gal ${ }^{+}$cells were counted in five fields. The number of $\beta$-gal ${ }^{+}$cells in control cultures treated with phosphate-buffered saline represents $100 \%$ survival. Data are averages from four independent experiments. Cells were cotransfected with PCMV- $\beta$-gal and APALAT (APALAT), PCMV$\beta$-gal and the empty expression plasmid instead of APALAT (PBABE Puro), or PCMV- $\beta$-gal and a plasmid expressing the baculovirus CPIAP (inhibitor of apoptosis) gene instead of APALAT (CPIAP). Similar results were obtained with a $L A T$ restriction fragment not containing a Poly(A) signal.

Table 2. Rabbits were infected and TGs harvested 7 days after infection. Five well-spaced sections were stained for apoptosis and examined from each of 10 TGs per group (50 sections per group), except for the uninfected control group, in which only 47 sections were examined.

\begin{tabular}{lcccc}
\hline \multicolumn{1}{c}{ Criterion } & dLAT2903 & dLAT2903R & McKrae & Control \\
\hline Sections with extensive apoptosis & $33 / 50(66 \%)$ & $2 / 50(4 \%)$ & $2 / 50(4 \%)$ & $2 / 47(4 \%)$ \\
TGs with extensive apoptosis & $9 / 10(90 \%)$ & $1 / 10(10 \%)$ & $2 / 10(20 \%)$ & $1 / 10(10 \%)$ \\
Sections with any apoptotic neurons & $37 / 50(74 \%)$ & $11 / 50(22 \%)$ & $8 / 50(16 \%)$ & $17 / 47(36 \%)$ \\
TGs with any apoptotic neurons & $10 / 10(100 \%)$ & $5 / 10(50 \%)$ & $5 / 10(50 \%)$ & $10 / 10(100 \%)$ \\
\hline
\end{tabular}

TGs on day 7 after infection.

To more directly test the hypothesis that $L A T$ has an antiapoptotic activity, we used a quantitative assay $(21,22)$ in which cells are cotransfected with a $\beta$-galactosidase expression plasmid ( $\mathrm{pCMV}-\beta$-gal) and an expression plasmid containing the gene of interest, in the presence of an inducer of apoptosis. If the gene of interest (in this case, $L A T$ ) reduces apoptosis, the number of $\beta$-gal ${ }^{+}$cells will be higher. Apoptosis was induced by the sphingoid base $\mathrm{C}_{6}$-ceramide (23-25), the mycotoxin fumonisin $\mathrm{B}_{1}\left(\mathrm{FB}_{1}\right)(26,27)$, or the anticancer drug etoposide. Primary human lung cells (IMR-90), monkey kidney cells (CV-1), and murine neuroblastoma cells (neuro-2A cells) were used for these studies.

An Apa I-Apa I LAT restriction fragment (nucleotides 301 to 2659 of the $8.3-\mathrm{kb}$ primary $L A T)$ containing the entire stable 2-kb $L A T$ region from HSV-1 strain KOS (5) was inserted into pBABE Puro, a mammalian expression vector containing a retrovirus long terminal repeat (LTR), polyadenylate [Poly(A)] addition signals, and the puromycin resistance gene regulated by the SV40 early promoter. The resulting plasmid (APALAT) was cotransfected into the respective cells along with pCMV- $\beta$-gal.
IMR-90 and CV-1 cells were subsequently treated with $\mathrm{C}_{6}$-ceramide or $\mathrm{FB}_{1}$ and neuro-2A cells with etoposide. Relative to cultures cotransfected with the empty pBABE Puro plasmid, the $L A T$-containing plasmid increased cell survival in all cultures (as judged by the number of $\beta$-gal ${ }^{+}$cells) regardless of the agent used to induce apoptosis (Fig. 3) $(P<0.0001$; ANOVA, Tukey posttest). Although the baculovirus antiapoptotic gene $C p I A P(28)$ appeared to inhibit apoptosis slightly more efficiently than did $L A T$, the differences were not significant $(P>0.05)$.

Ceramide-induced apoptosis is blocked by activating protein kinase $\mathrm{C}(23,25)$, whereas blocking the tumor necrosis factor pathway (TNF/FAS) inhibits $\mathrm{FB}_{1}$-induced apoptosis (26, 29). Etoposide inhibits topoisomerase II, thus leading to higher levels of DNA damage and p53-dependent apoptosis $(13,14,30)$. The ability of $L A T$ to block apoptosis induced by each of these agents therefore suggests that $L A T$ interferes with a downstream effector of apoptosis that is common to many apoptotic pathways. In addition, the ability of the APALAT fragment to block apoptosis mapped this function to within a region comprising only $28 \%$ of the primary $L A T$. The ability of this region to promote efficient establishment and subsequent reactivation from latency (5) strengthens the likelihood that $L A T$ 's antiapoptosis function plays an important role in the latency reactivation cycle. The antiapoptotic activity of $L A T$ did not appear to be HSV-1 strain specific, because we saw this activity with strain McKraederived viruses and strain KOS LAT.

Previous studies have reported that under different conditions, herpes simplex virus can induce or inhibit apoptosis $(15,31,32)$. At least two other viral genes, ICP27 and US3, can protect certain cells against virus-induced apoptosis in tissue culture $(31,32)$. Our results show that $L A T$ can suppress apoptosis of neurons (either in vivo or in vitro) and that this function may explain the importance of $L A T$ in herpes simplex virus latency and reactivation. Interestingly, we recently found that the latency-related $(L R)$ gene of bovine herpes virus (BHV-1) also inhibits apoptosis in transient transfection assays (33). BHV-1 and HSV-1 are only distantly related. The 


\section{R E P O R T S}

BHV-1 LR and the HSV-1 LAT have greatly different sizes and little or no sequence similarity. Although the BHV-1 LR antiapoptosis function correlates with expression of an LR protein (33), the $L A T$ antiapoptosis function may be mediated by the LAT RNA, because $L A T$ does not appear to encode a protein (34).

Our results suggest that $L A T$ promotes neuronal survival after HSV-1 infection by reducing apoptosis. This hypothesis is supported by studies indicating that $L A T^{-}$mutants establish latency less efficiently than does the wild type (5, $6,35,36)$ and that a mutant expressing an altered LAT has increased neurovirulence (12). In general, stress is associated with reactivation and increased corticosteroid levels. Because corticosteroids induce apoptosis $(37,38)$, stress and viral gene expression during reactivation may induce apoptosis. Because $L A T$ facilitates reactivation $(3,39,40)$, inhibition of apoptosis by $L A T$ would increase the probability that productive infection (i.e., productive reactivation from latency) succeeds. Whether reactivation was successful or not, $L A T^{+}$neurons may have a better chance to survive and resume latency. Thus, a $L A T$ antiapoptosis function could allow $L A T$ to enhance reactivation by (i) enhancing the establishment and maintenance of latency, thereby providing more latently infected neurons in which future reactivations could occur; (ii) facilitating productive reactivation by protecting against apoptosis in neurons in which reactivation occurs; and (iii) facilitating the resumption of latency after a reactivation insult by protecting neurons against apoptosis.

It is unlikely that $L A T$ is the only factor that promotes neuronal survival, because terminally differentiated neurons must have a well-devised mechanism to prevent programmed cell death. Furthermore, LAT may have additional mechanisms by which it enhances reactivation. Nonetheless, our results strongly suggest that suppressing apoptosis is an important mechanism by which $L A T$ enhances HSV-1 reactivation. In addition, the ability of $L A T$ to prevent HSV-1-induced apoptosis may be important in preventing the virus from causing extensive neuronal damage and subsequent neuronal disorders.

\section{References and Notes}

1. D. L. Rock et al., J. Virol. 61, 3820 (1987).

2. J. G. Stevens, E. K. Wagner, G. B. Devi-Rao, M. L. Cook L. T. Feldman, Science 235, 1056 (1987).

3. G. C. Perng et al., J. Virol. 68, 8045 (1994).

4. G. C. Perng, H. Ghiasi, S. M. Slanina, A. B. Nesburn, S. L. Wechsler, J. Virol. 70, 976 (1996).

5. R. L. Thompson and N. M. Sawtell, J. Virol. 71, 5432 (1997).

6. N. M. Sawtell, J. Virol. 71, 5423 (1997)

7. , J. Virol. 72, 6888 (1998).

8. , D. K. Poon, C. S. Tansky, R. L. Thompson, J. Virol. 72, 5343 (1998)

9. Y. J. Gordon, E. G. Romanowski, T. Araullo-Cruz, P. R. Kinchington, Graefes Arch. Clin. Exp. Ophthalmol. 233, 649 (1995).

10. N. Mador, D. Goldenberg, O. Cohen, A. Panet, I. Steiner, J. Virol. 72, 5067 (1998). U.S.A. 95, 3931 (1998).

24. R. Bose et al., Cell 82, 405 (1995).
11. D. A. Garber, P. A. Schaffer, D. M. Knipe, J. Virol. 71, 5885 (1997).

12. G. C. Perng et al., J. Virol. 73, 920 (1999).

13. J. Nip et al., Mol. Cell. Biol. 17, 1049 (1997).

14. J. G. Boesen-de Cock, A. D. Tepper, E. de Vries, W. J van Blitterswijk, J. Borst, J. Biol. Chem. 274, 14255 (1999)

15. V. Galvan and B. Roizman, Proc. Natl. Acad. Sci.

16. V. Galvan, R. Brandimarti, B. Roizman, J. Virol. 73, 3219 (1999)

17. G.-C. Perng, C. Jones, J. Ciacci-Zanella, M. Stone, G. Henderson, A. Yukht, S. M. Slanina, F. M. Hofman, H. Ghiasi, A. B. Nesburn, S. L. Wechsler, data not shown.

18. P. J. Duriez and G. M. Shah, Biochem. Cell. Biol. 75 337 (1997).

19. C. M. Simbulan-Rosenthal, D. S. Rosenthal, S. Iyer, A. H. Boulares, M. E. Smulson, J. Biol. Chem. 273, 13703 (1998).

20. J. Saldeen and N. Welsh, Mol. Cell. Endocrinol. 139, 99 (1998).

21. S. Kumar, M. Kinoshita, M. Noda, N. G. Copeland, N. A. Jenkins, Genes Dev. 8, 1613 (1994).

22. H. Hsu, J. Xiong, D. V. Goeddel, Cell 81, 495 (1995).

23. O. Cuvillier et al., Nature 381, 800 (1996).

25. L. M. Obeid, C. M. Linardic, L. A. Karolak, Y. A. Hannun, Science 259, 1769 (1993)

26. J. Ciacci-Zanella and C. Jones, Food Chem. Toxicol. 37, 703 (1999).

27. W. Wang et al., Proc. Natl. Acad. Sci. U.S.A. 93, 3461 (1996)
28. R. J. Clem and L. K. Miller, Mol. Cell. Biol. 14, 5212 (1994).

29. C. Jones, J. R. Ciacci-Zanella, Y. Zhang, G. Henderson, M. M. Dickman, Environ. Health Perspect., in press.

30. J. D. Hainsworth and F. A. Greco, Ann. Oncol. 6, 325 (1995).

31. M. Aubert and J. A. Blaho, J. Virol. 73, 2803 (1999).

32. R. Leopardi, C. Van Sant, B. Roizman, Proc. Natl. Acad. Sci. U.S.A. 94, 7891 (1997).

33. J. Ciacci-Zanella, M. Stone, G. Henderson, C. Jones, J. Virol. 73, 9734 (1999)

34. B. S. Drolet et al., Virology 242, 221 (1998).

35. N. M. Sawtell and R. L. Thompson, J. Virol. 66, 2157 (1992).

36. G. C. Perng et al., J. Virol. 74, 1885 (2000).

37. E. S. Dieken and R. L. Miesfeld, Mol. Cell. Biol. 12, 589 (1992).

38. I. J. Mitchell, A. J. Cooper, M. R. Griffiths, D. J. Barber, Neuroscience 84, 489 (1998).

39. C. Jones, Adv. Virus Res. 51, 81 (1998).

40. E. K. Wagner and D. C. Bloom, Clin. Microbiol. Rev. 10, 419 (1997).

41. We thank L. Miller for providing the plasmid containing CPIAP, R. Eppley for $\mathrm{FB}_{1}$ (>99.9\% pure), and R. Thompson for APALAT. Supported by Public Health Service grants EY07566 and EY11629, the Discovery Fund for Eye Research, The Skirball Program in Molecular Ophthalmology, the Center for Biotechnology, UNL, the Comparative Pathobiology area of concentration, USDA grants 9702394 and 9802064, and a scholarship from CNPq, Brazil (J.C.-Z.)

28 June 1999; accepted 7 January 2000 